\title{
The difference between observed and expected prevalence of MCAD deficiency in The Netherlands: a genetic epidemiological study
}

\author{
Terry GJ Derks ${ }^{*}{ }^{1}$, Marinus Duran ${ }^{2}$, Hans R Waterham ${ }^{2}$, Dirk-Jan Reijngoud ${ }^{1}$, Leo P ten Kate ${ }^{3}$ \\ and G Peter A Smit ${ }^{1}$ \\ ${ }^{1}$ Department of Metabolic Diseases, Beatrix Children's Hospital, University Medical Centre, Groningen, The \\ Netherlands; ${ }^{2}$ Laboratory Genetic Metabolic Diseases, Department of Clinical Chemistry, Academic Medical Centre \\ Amsterdam, Amsterdam, The Netherlands; ${ }^{3}$ Department of Clinical Genetics and Human Genetics, VU University \\ Medical Center, Amsterdam, The Netherlands
}

Medium chain acyl coenzyme A dehydrogenase (MCAD) deficiency is assumed to be the most common inherited disorder of mitochondrial fatty acid oxidation. Few reports mention the difference between the expected and observed prevalence of MCAD deficiency on the basis of the carrier frequency in the population. We performed a population-wide retrospective analysis of all known MCAD-deficient patients in The Netherlands. In this study, the observed prevalence of MCAD deficiency in The Netherlands was $1 / 27400$ (95\% confidence interval $(\mathrm{Cl}) 1 / 23000-1 / 33900)$, significantly different from the expected prevalence of $1 / 12100(95 \% \mathrm{Cl} 1 / 8450-1 / 18500)$. The observed prevalence of MCAD deficiency showed a remarkable north-south trend within the country. From the patients in this cohort, it can be observed that underdiagnosis contributes to a larger extent to the difference between the expected and observed prevalences of MCAD deficiency in our country, than reduced penetrance. We determined estimates of the segregation proportion in a cohort of 73 families under the assumption of complete ascertainment $\left(p_{\mathrm{LM}}=0.41,95 \% \mathrm{Cl} 0.31-0.51\right)$ and single ascertainment $\left(p_{\mathrm{D}}=0.28,95 \% \mathrm{Cl} 0.19-0.37\right)$. With the expectation-maximization algorithm, a third estimate was obtained $\left(p_{\mathrm{EM}}=0.28,95 \% \mathrm{Cl} 0.20-0.37\right)$. The agreement between the latter two estimates supports incomplete selection and the segregation proportions were in agreement with normal mendelian autosomal recessive inheritance. European Journal of Human Genetics (2005) 13, 947-952. doi:10.1038/sj.ejhg.5201428; published online 4 May 2005

Keywords: segregation analysis; prevalence; north-south trend; The Netherlands; fatty acid oxidation; MCAD deficiency

Introduction

Medium chain acyl coenzyme A dehydrogenase (MCAD [E.C.1.3.99.3; MIM 201450]) deficiency is assumed to be

*Correspondence: Mr TGJ Derks, Department of Metabolic Diseases, Beatrix Children's Hospital, University Medical Centre, Groningen, PO Box 30 001, 9700 RB Groningen, The Netherlands. Tel: + 3150 3614147; Fax: + 3150 3614235; E-mail: t.g.j.derks@bkk.umcg.nl Received 5 January 2005; revised 21 March 2005; accepted 24 March 2005; published online 4 May 2005 the most common inherited disorder of fatty acid oxidation. ${ }^{1}$ In the classic clinical presentation, fasting precipitates acute symptoms in early infancy or childhood, mostly in combination with an increased energy need during simple infections. ${ }^{2,3}$ Patients develop drowsiness or lethargy, which may proceed into coma or even sudden death. Biochemically, the disease is characterized by a hypoketotic hypoglycaemia and specific metabolites can be detected in plasma and urine. The point mutation A to 
$\mathrm{G}$ at nucleotide 985 of the $M C A D$ gene is found in approximately $90 \%$ of mutant alleles, of patients identified after clinical expression of the disorder. ${ }^{4-6}$

In several populations, the $985 \mathrm{G}>\mathrm{A}$ carrier frequency is determined (for an overview, see Wang et $a l^{7}$ ) and from these studies it can be observed that the distribution pattern of this mutation shows a high prevalence in many eastern and northern countries in Europe. Lower frequencies are observed in the southern part of western and middle Europe. ${ }^{8}$ The prevalence of carriers of the $985 \mathrm{G}>\mathrm{A}$ mutation in The Netherlands was previously determined to be 1/59 (95\% confidence interval (CI) 1/50-1/73), while the relative frequency of the $985 \mathrm{G}>\mathrm{A}$ mutation was $94 \%$ in 47 patients. Hence, the expected prevalence of MCAD deficiency among newborns in our country would be 1/12 100 (95\% CI 1/8450-1/18500) under Hardy-Weinberg conditions. ${ }^{9}$ The Netherlands has about 16 million inhabitants with more that 200000 newborns each year, thus about $16(10-23)$ new cases are expected annually (CBS, Dutch Central Bureau for Statistics).

Previous studies have shown that the observed prevalence of patients with MCAD deficiency may be lower than the prevalence one must expect on the basis of observed carrier frequencies. ${ }^{10,11}$ However, in these reports, possible explanations for this observation have not been investigated. Theoretically, underreporting or underdiagnosis, reduced penetrance with a lifelong clinical tolerance for the enzyme disorder, prenatal loss of affected offspring or abnormal segregation of alleles in heterozygous parents could explain this discrepancy. Case reports describing asymptomatic family members illustrate the possibility of reduced penetrance, ${ }^{12}$ and misdiagnosis after expression of the disorder is a well-known phenomenon also. ${ }^{3}$ However, a study in a large well-defined population has not been carried out yet. Despite this lack of knowledge, currently MCAD deficiency is under evaluation for population newborn screening in several areas.

Knowledge about the prevalence of a disorder is of great importance for decision-making about population screening. ${ }^{13}$ Therefore, a population-wide retrospective analysis was performed of all known MCAD-deficient patients in The Netherlands. In this study, the observed prevalence of MCAD deficiency in The Netherlands was compared with the expected prevalence among newborns based on previously found allele frequencies. From the clinical information and family characteristics of MCAD-deficient patients, possible explanations for the discrepancy between the expected and observed prevalence of MCAD deficiency are investigated.

\section{Methods \\ Patients}

In The Netherlands, medical care for patients with metabolic defects is restricted to several metabolic subdivi- sions of pediatric departments in the eight university hospitals. Only their affiliated laboratories perform specific metabolic diagnostic procedures. In order to facilitate a population-wide analysis of MCAD-deficient patients in The Netherlands, we contacted these metabolic departments to gain insight into the medical records of the known families with MCAD deficiency. Data were complemented with the data of the affiliated metabolic laboratories and the three clinical genetic laboratories, which had performed the specific MCAD DNA analysis in The Netherlands. Data of patients born before July 2003 were documented in Case Record Forms (CRF), one for each patient. In the CRF, data were recorded concerning clinical history, laboratory data, family history, place of residence at the time of diagnosis and data on follow-up after diagnose.

Families were ascertained through probands who had a clinical expression of the disorder and were diagnosed initially. Screening of family members had been performed in most cases including parents and siblings. After discussion with the responsible clinicians from each family, the clinical history could be reconstructed and the proband could be identified. No siblings were identified as patients independently. Patients were regarded to have MCAD deficiency, when an enzyme assay, DNA analysis or a combination had confirmed the diagnosis. Few patients were included also, if and only if an enzyme assay and/or DNA analysis in family members had confirmed the diagnosis, when clinical history or standard metabolic diagnostic procedures pointed strongly into the direction of MCAD deficiency, that is, a hypoketotic hypoglycaemia combined with the specific patterns in the measurements of urine organic acids and/or plasma acylcarnitines. Patients were categorized according to their clinical manifestation as classical proband, symptomatic sibling, clinically asymptomatic sibling or neonatally detected sibling. Data from the CRFs were anonymously archived in a database and retrieved before data analysis.

\section{Data analysis}

The prevalence $P$ of MCAD deficiency was determined by dividing the total number of cases of MCAD deficiency by the total number of newborns. The formula used for calculation of the CI of the prevalence was as follows:

$$
95 \% \mathrm{CI}=P \pm 1,96 \times((P \times(1-P)) / N)^{1 / 2}
$$

where $N$ is the number of living newborns.

Using data from the Dutch Central Bureau for Statistics (Centraal Bureau voor de Statistiek (CBS), www.cbs.nl), prevalence was calculated for the country as a whole and for different subdivisions. The Netherlands consists of 12 provinces which were assigned a number as follows: the province of Friesland 1, Groningen 2, Drenthe 3, Overijssel 4, Gelderland 5, Flevoland 6, Utrecht 7, NoordHolland 8, Zuid-Holland 9, Zeeland 10, Noord-Brabant 11 
and Limburg 12. Subsequently, two ways of subdividing the country were chosen. First, a historical/geographic division was used by combining the provinces in three areas, taking into account the natural barriers in our country: the former Zuiderzee and the major branches of the rivers Rhine and Meuse, as performed previously. ${ }^{14}$ Area A consists of the provinces numbered 1-6, area B includes numbers 7-9, while area $C$ includes the remaining three southern provinces. Secondly, subdivision according to the regions corresponding to the organization of the Dutch neonatal screening program was used, resulting in five regions. Region Northeast (NE) contains the provinces numbered 1-4, region Middle (Mid) includes 5 and 7, region Northwest (NW) is formed by numbers 6 and 8 , region Southwest (SW) by numbers 9 and 10, while region Southeast (SE) includes provinces 11 and 12 .

\section{Segregation analysis}

Although the family data already showed that there was a low number of probands per family, we performed three types of analysis, one assuming truncate selection, one assuming single selection and another without prior assumption on the type of ascertainment. For the case of truncate selection (complete ascertainment: all affected siblings are identified as probands independently), the $\mathrm{Li}$ and Mantel estimator for segregation proportion was calculated as:

$$
p_{\mathrm{LM}}=\left(R-J_{1}\right) /\left(T-J_{1}\right)
$$

where $R$ is the total number of affected children, $J_{1}$ is the total number of families with a single affected child and $T$ is the total number of offspring in the sample. Weight tables by $\mathrm{Li}$ and Mantel were used to compute the variance and standard error. The $95 \%$ CI was ${ }^{15}$

$$
p_{\mathrm{LM}}=p_{\mathrm{LM}} \pm 1.96 \times S E
$$

For the case of single selection (low probability of ascertainment, families have only one proband), a modified $\mathrm{Li}$ and Mantel approach proposed by Davie was applied. The Davie estimator for segregation analysis $\left(p_{\mathrm{D}}\right)$ was calculated as:

$$
p_{\mathrm{D}}=(R-J) /(T-J)
$$

where $R$ is the total number of affected children, $J$ is the total number of families with a single proband and $T$ is the total number of offspring in the sample. The variance of $p_{D}$ can be approached by: ${ }^{16}$

$$
(R-J)(T-R) /(T-J)
$$

For the case of multiple incomplete ascertainment, the expectation-maximization (EM) algorithm is used to compute the estimate of the segregation proportion $\left(p_{\mathrm{EM}}\right){ }^{17}$ Intervals for the EM estimate of the segregation proportion are obtained by a bootstrapping method and a $95 \%$ bootstrap interval estimate is computed by taking the 2.5th and 97.5th percentiles of the bootstrap distribution. ${ }^{18}$

\section{Statistics}

Differences between groups of patients were analysed using nonparametric tests.

\section{Results \\ Patients}

In The Netherlands, 110 unrelated families containing 155 patients with MCAD deficiency were found. In each family the proband was identified and in addition there were 10 symptomatic siblings, 17 clinically asymptomatic siblings and 18 siblings diagnosed in the newborn period. In Figure 1, the numbers of patients with MCAD deficiency from the birth cohorts 1980-1999 are plotted according to this clinical presentation. Comparing the cohort '80-'89 and '90-'99, the observed prevalences in these periods were $1 / 30500$ (95\% CI 1/24 400-1/41 000) and $1 / 28400$ (95\% CI 1/22900-1/37 100), respectively. The number of asymptomatic siblings decreased significantly with nine children who were clinically asymptomatic in the 80th decade and only two such children in the 90th decade (Fisher Exact, $P=0.02$ ). At the same time, the number of patients identified as neonate showed an increasing trend (Fisher Exact, $P=0.05$ ). Twenty-seven children in total suffered a fatal manifestation, nine of whom were not diagnosed initially but only after diagnosing the proband in the family. Subsequent to the diagnosis no mortality was observed, except in one girl with additionally Rett syndrome. No significant decrease in mortality due to MCAD deficiency was seen comparing the two decades.

Excluding the neonates diagnosed prospectively, 25 patients (18\%) had one or more clinical admission before diagnosis was made. These children were equally distributed between the birth cohorts. However, children born in the 70 s and 80 s represented cases with relatively more admissions before diagnosis.

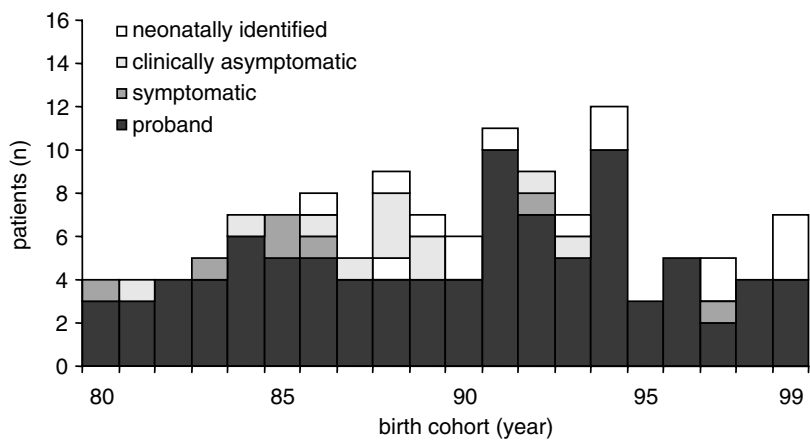

Figure 1 Number of patients with MCAD deficiency in the birth cohorts 1980-1999 according to their mode of clinical manifestation. 
Table 1 Expected and clinical prevalence of MCAD deficiency in the Netherlands

\begin{tabular}{|c|c|}
\hline MCAD deficiency in the Netherlands & Frequencies \\
\hline $\begin{array}{l}\text { Guthrie cards tested }^{\mathrm{a}} \\
985 \mathrm{G}>\mathrm{A} \text { heterozygotes } \\
{ }^{\mathrm{a}} \\
\text { Observed relative } 985 \mathrm{C}>\mathrm{A} \text { allele frequency } \mathrm{y}^{\mathrm{a}, \mathrm{b}} \\
\text { MCAD mutation carrier frequency }(95 \% \mathrm{Cl})^{\mathrm{a}} \\
\text { Expected prevalence of MCAD deficiency }(95 \% \mathrm{Cl})^{\mathrm{a}} \\
\text { Newborns } 1985-1999^{\mathrm{c}} \\
\text { Expected number of patients ' } 85-^{\prime} 99(\text { range) } \\
\text { Observed number of patients ' } 85-^{\prime} 99^{\mathrm{b}} \\
\text { Prevalence of detected patients }(95 \% \mathrm{Cl}) \\
\text { Observed/expected ratio (range) }\end{array}$ & $\begin{array}{c}6195 \\
99 \\
94 \% \\
1 / 55(1 / 46-1 / 68) \\
1 / 12100(1 / 8450-1 / 18500) \\
2879529 \\
238(156-341) \\
105 \\
1 / 27400(1 / 23000-1 / 33900) \\
44 \%(31-67)\end{array}$ \\
\hline
\end{tabular}

${ }^{a}$ According to De Vries et al. ${ }^{9}$

${ }^{\mathrm{b}}$ Current study.

${ }^{\mathrm{C}}$ According to CBS.

\section{Observed prevalence of MCAD deficiency in The Netherlands}

The first clinically oriented Dutch reports describing MCAD deficiency originate from 1985 and we assume that MCAD deficiency has been a relatively known disease entity in academic hospitals within The Netherlands since then. As, in our cohort studied, initial acute manifestations occurred between 0 days and 5.1 years, the prevalence of MCAD deficiency in our country was calculated including only patients born between 1985 and 1999. In this period we identified 105 patients and therefore the observed prevalence was $1 / 27400$ (95\% CI 1/23000-1/33900) (Table 1). In the families from this cohort, the $985 \mathrm{G}>\mathrm{A}$ allele frequency was $94 \%$, which is in agreement with the estimate previously used in calculating the estimated prevalence. $^{9}$

\section{Regional differences of MCAD deficiency prevalence in The Netherlands}

The regional prevalences of MCAD deficiency in The Netherlands are shown in Figure 2. Significant differences were found between the three geographic/historical regions $\left(\chi^{2}=14.4, P=0.001\right.$ at $\left.2 \mathrm{df}\right)$. The prevalence in region A was significantly higher than in the rest of the country $\left(\chi^{2}=12.9, P<0.001\right.$ at $\left.1 \mathrm{df}\right)$ and when region $\mathrm{A}+\mathrm{B}$ was compared with region $\mathrm{C}$ a significant difference could be demonstrated still $\left(\chi^{2}=6.1, P<0.05\right.$ at $\left.1 \mathrm{df}\right)$, reflecting a north-south gradient in The Netherlands.

When the prevalences of MCAD deficiency were compared between the regions corresponding to the organization of the Dutch neonatal screening program, significant differences were observed also $\left(\chi^{2}=17.1, P<0.01\right.$ at $\left.4 \mathrm{df}\right)$. The highest prevalence was found in region NE, namely 1/16000 (95\% CI 1/11 800-1/24 700).

\section{Segregation analysis}

Data from the families diagnosed between 1985 and 1999 were used assuming that family members were investigated routinely and excluding the youngest (incomplete) fa-
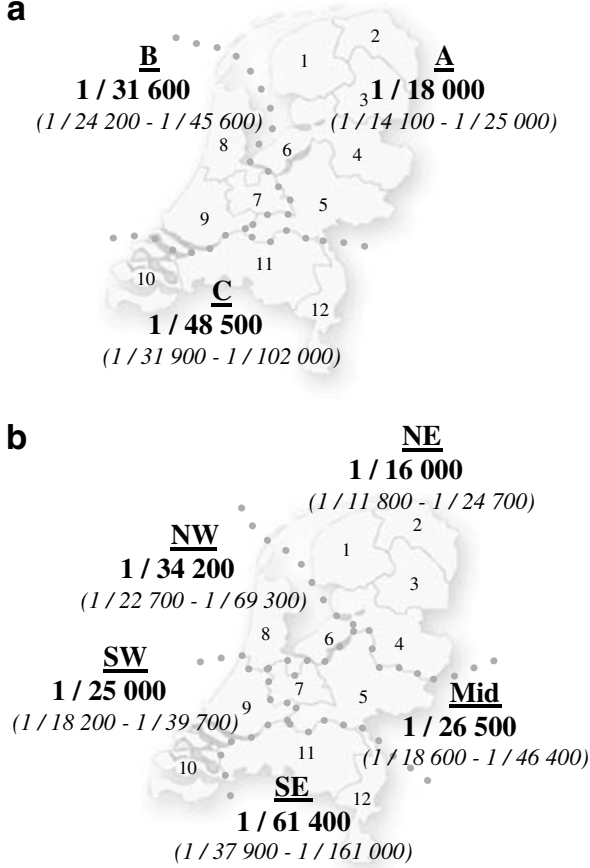

Figure 2 Regional variations in observed prevalence of MCAD deficiency in The Netherlands. The country is divided into three areas based on historical/geographic barriers (a), as well as into the screening areas (b) as described in 'Methods'.

milies. Families with incomplete data and one family with an affected father were excluded also. Table 2 shows the characteristics of included families. The observed proportion of affected children calculated was 0.54 (instead of the expected 0.25 under complete selection). The estimate for the segregation proportion for truncate selection was $p_{\mathrm{LM}}=0.41(95 \%$ CI $0.31-0.51)$ and for single selection $p_{\mathrm{D}}=0.28$ (95\% CI 0.19-0.37). Under multiple incomplete ascertainment, the estimate was computed $p_{\mathrm{EM}}=0.28(95 \%$ CI 0.20-0.37). 
Table 2 Segregation analysis for MCAD deficiency

\begin{tabular}{lrrrrrrr} 
Affected sibs & \multicolumn{1}{c}{ Families } & \multicolumn{1}{c}{ Number of families by number of sibs } \\
per family $(n)$ & $(n)$ & 1 & 2 & 3 & 4 & 5 & 8 \\
\hline 1 & 46 & 6 & 27 & 8 & 5 & 0 & 0 \\
2 & 20 & 0 & 4 & 9 & 4 & 2 & 1 \\
3 & 5 & 0 & 0 & 2 & 1 & 2 & 0 \\
4 & 2 & 0 & 0 & 0 & 2 & 0 & 0 \\
Total & 73 & 6 & 31 & 19 & 12 & 4 & 1 \\
\hline
\end{tabular}

From the birth cohorts 1985-1999, we included 73 families $(J)$ with 109 affected $(R)$ children. The total number of offspring is $201(T)$. The number of families with one affected child was $49\left(J_{1}\right)$.

\section{Discussion}

In this study only directly or indirectly (through family members) diagnosed cases could be included, so no direct information about the cases that remain undiagnosed outside these families was available. Nevertheless, from a well-defined population with complete ascertainment of diagnosed cases, lessons of great importance can be drawn.

The observed prevalence of MCAD deficiency in The Netherlands was $1 / 27400$ (95\% CI 1/23000-1/33900), significantly lower than expected, and the $985 \mathrm{G}>$ A allele frequency was $94 \%$, as was previously used in calculating the estimated prevalence. Fromenty $e t a l^{10}$ already noticed a discrepancy between the number of expected and observed cases of MCAD deficiency in their study. They used the $985 \mathrm{G}>\mathrm{A}$-carrier frequency obtained from 414 French Caucasian blood donor volunteers from the Paris area to calculate the expected frequency of homozyotes for the whole of France. This approach is questionable as the 985G $>$ A-carrier frequency within France decreases in southerly direction. ${ }^{8}$ Unfortunately, no clinical background information was provided about their diagnosed cases. In our cohort underdiagnosis (25 cases suffered clinical admissions before diagnosis, nine children were initially not diagnosed after a fatal presentation) contributed to a larger extent than a reduced penetrance (17 clinically asymptomatic sibling), in explaining this discrepancy.

The data concerning the clinical prevalence of MCAD deficiency in The Netherlands clearly show a north-south gradient. In The Netherlands, the $985 \mathrm{G}>\mathrm{A}$ carrier frequency was determined previously to be $1 / 59$ (95\% CI $1 / 50-1 / 73) .{ }^{9}$ Compared to neighbouring countries and areas (Belgium 1/77, Germany 1/116, Normandy 1/118, and the prevalence decreased in southern parts of Western Europe ${ }^{8}$ for an overview, see Wang $e t a l^{7}$ ), the frequency in our country was rather high. Unfortunately, the regional distribution of the $985 \mathrm{G}>\mathrm{A}$ frequency in The Netherlands was not assessed in the previous study. However, in our calculations we assumed that the relative frequency of $985 \mathrm{G}>\mathrm{A}$ did not differ between regions. Although this is an unproven assumption, it would mean that we should postulate a north-south gradient of the $985 \mathrm{G}>$ A mutation. Probably, former historical/geographic barriers played an important role in this development. This issue of a historical/geographic trend in allele frequency has been raised before in the distribution of mutations of cystic fibrosis mutations in our country. ${ }^{14}$ At the other side, Seddon et al $^{11}$ already showed, that in a region where clinicians are expected to have a high awareness of MCAD deficiency, the expected number of cases could be diagnosed. In The Netherlands, the locations of academic hospitals are distributed proportionally to the number of inhabitants, but, obviously, mostly acutely presenting patients are visiting general medical doctors instead of doctors with specific metabolic interest. So it remains speculative whether differences in awareness could be an additional explanation for the differences in clinical prevalence in The Netherlands.

Segregation analysis has not been performed for MCAD deficiency before and aberrant results could reveal an additional explanation for underdiagnosis of MCAD deficiency. A rationale for postulating abnormal segregation of alleles can be obtained from both animal and human studies, in which the importances of mitochondrial fatty acid oxidation and carnitine for spermatogenesis are illustrated. ${ }^{19-21}$ The estimates for the segregation proportion under single selection and multiple incomplete ascertainment were in agreement with normal unselective mendelian autosomal recessive inheritance of MCAD deficiency, as the interval estimates included the value $P=0.25$. The close resemblance between $p_{\mathrm{D}}$ and $p_{\mathrm{EM}}$ suggests that in this study an assumption of complete ascertainment of affected patients was not warranted. Therefore, a selection of alleles is not needed to explain the lower observed prevalence of MCAD deficiency in this population.

In conclusion, the results of this study show that in The Netherlands the diagnosed number of patients with MCAD deficiency is significantly lower than the expected number. This discrepancy is mainly explained by misdiagnosis but reduced penetrance is an important factor also. No significant decrease of the number of fatal presentations is observed and it is assumable that the observed annual number of 1-2 deceased children is an underestimate. A regional difference in observed prevalence of MCAD deficiency is found, probably due to a north-south gradient of the $985 \mathrm{G}>\mathrm{A}$ mutation in The Netherlands. Segregation analysis in MCAD deficiency does not give another explanation for the difference between estimated prevalence and observed prevalence.

\section{Acknowledgements}

We thank all metabolic and general pediatricians and their cooperating departments and laboratories in The Netherlands for 
providing insight into the patient records. We thank HJ Stellingwerff for performing the EM algorithm. ZonMw supported this study financially.

\section{References}

1 Roe CR, Ding J: Mitochondrial fatty acid oxidation disorders; in Scriver CR, Beaudet AL, Sly WS, Valle D (eds): The Metabolic \& Molecular Bases of Inherited Disease. McGraw-Hill Medical Publishing Division: New York. Vol 8. 2001, pp 22972326.

2 Touma EH, Charpentier C: Medium chain acyl-CoA dehydrogenase deficiency. Arch Dis Child 1992; 67: 142-145.

3 Iafolla AK, Thompson Jr RJ, Roe CR: Medium-chain acylcoenzyme A dehydrogenase deficiency: clinical course in 120 affected children. J Pediatr 1994; 124: 409-415.

4 Gregersen N, Blakemore AI, Winter V et al: Specific diagnosis of medium-chain Acyl-CoA dehydrogenase MCAD deficiency in dried blood spots by a polymerase chain reaction PCR assay detecting a point-mutation G985 in the MCAD gene. Clin Chim Acta 1991; 203: 23-34.

5 Yokota I, Coates PM, Hale DE, Rinaldo P, Tanaka K: Molecular survey of a prevalent mutation, 985A-to-G transition, and identification of five infrequent mutations in the medium-chain Acyl-CoA dehydrogenase (MCAD) gene in 55 patients with MCAD deficiency. Am J Hum Genet 1991; 49: $1280-1291$.

6 Tanaka K, Yokota I, Coates PM et al: Mutations in the medium chain acyl-CoA dehydrogenase (MCAD) gene. Hum Mutat 1992; 1: 271-279.

7 Wang SS, Fernhoff PM, Hannon WH, Khoury MJ: Medium chain acyl-CoA dehydrogenase deficiency human genome epidemiology review. Genet Med 1999; 1: 332-339.

8 Tanaka K, Gregersen N, Ribes A et al: A survey of the newborn populations in Belgium, Germany, Poland, Czech Republic, Hungary, Bulgaria, Spain, Turkey, and Japan for the G985 variant allele with haplotype analysis at the medium chain acyl-CoA dehydrogenase gene locus: clinical and evolutionary consideration. Pediatr Res 1997; 41: 201-209.
9 De Vries HG, Niezen-Koning KE, Kliphuis JW et al: Prevalence of carriers of the most common medium-chain acyl-CoA dehydrogenase (MCAD) deficiency mutation (G985A) in the Netherlands. Hum Genet 1996; 98: 1-2.

10 Fromenty B, Mansouri A, Bonnefont JP et al: Most cases of medium-chain acyl-CoA dehydrogenase deficiency escape detection in France. Hum Genet 1996; 7: 367-368.

11 Seddon HR, Green A, Gray RG, Leonard JV, Pollitt RJ: Regional variations in medium-chain acyl-CoA dehydrogenase deficiency. Lancet 1995; 345: 135-136.

12 Duran M, Hofkamp M, Rhead WJ, Saudubray JM, Wadman SK: Sudden child death and 'healthy' affected family members with medium-chain acyl-coenzyme A dehydrogenase deficiency. Pediatrics 1986; 78: 1052-1057.

13 Wilson JM, Jungner YG: Principios y metodos del examen colectivo para identificar enfermedades. Bol Oficina Sanit Panam 1968; 65: 281-393.

14 Collee JM, De Vries H, Scheffer H, Halley D, Ten Kate L: Relative frequencies of cystic fibrosis mutations in The Netherlands, as an illustration of significant regional variation in a small country. Hum Genet 1998; 102: 587-590.

15 Li CC, Mantel N: A simple method of estimating the segregation ratio under complete ascertainment. Am J Hum Genet 1968; 20: $61-81$

16 Davie AM: The 'singles' method for segregation analysis under incomplete ascertainment. Ann Hum Genet 1979; 42: 507-512.

17 Lange K: Mathematical and Statistical Methods for Genetic Analysis. New York: Springer, 1997.

18 Efron BTR: An Introduction to Bootstrap. New York: Chapman and Hall, 1993.

19 Casillas ER: Accumulation of carnitine by bovine spermatozoa during maturation in the epididymis. J Biol Chem 1973; 248: $8227-8232$.

20 Toshimori K, Kuwajima M, Yoshinaga K, Wakayama T, Shima K: Dysfunctions of the epididymis as a result of primary carnitine deficiency in juvenile visceral steatosis mice. FEBS Lett 1999; 446: $323-326$.

21 Xuan W, Lamhonwah AM, Librach C, Jarvi K, Tein I: Characterization of organic cation/carnitine transporter family in human sperm. Biochem Biophys Res Commun 2003; 306: $121-128$. 